\title{
Image Watermarking using DC Component of DCT
}

\author{
Rifat Kurban \\ Dept. of Computer Engineering \\ Erciyes University \\ Kayseri, Turkey \\ rkurban@erciyes.edu.tr
}

\author{
Hakki Bozpolat \\ Informatics and Inf. Security Research Center \\ TUBITAK \\ Gebze, Turkey \\ hakki.bozpolat@tubitak.gov.tr
}

\author{
Florenc Skuka \\ Dept. of Computer Engineering \\ Erciyes University \\ Kayseri, Turkey \\ skuka.f@gmail.com
}

\begin{abstract}
In this paper, a robust watermarking method based on modifying the DC component of the DCT coefficient is presented. A random generated key sequence is embedded into the DC coefficients of $8 \times 8$ blocks with a scaling factor. Effect of the scaling factor under several attacks is evaluated. Experiments show that the method is robust without losing the transparency.
\end{abstract}

Keywords - Image watermarking, Robust watermarking, DCT, DC Component

\section{INTRODUCTION}

The acquisition and processing of digital information has become widespread in the recent years as a result of the increase in the use of internet both in terms of the number of users and the link speed. Consequently, the need has emerged for the protection of digital pictures against copying. One of the main methods which have been proposed for the protection of copyright is the watermarking of digital images [1].

Digital watermarking is defined as the hiding of digital data inside another digital data as a seal. Digital watermarking methods may be used for many different purposes including the protection of copyrights, data confirmation and data hiding [2]. Two different kinds of watermarking operations are implemented on digital information namely visible and invisible. In visible watermarking, the watermark is placed on an area of digital image in a way to be detected visually. In invisible watermarking, the watermark is hidden and can only be extracted by the person who embeds it with a definite algorithm [3].

Watermarking techniques are categorized into two as spatial and transform domain techniques according to the domain in which the watermark will be embedded. Spatial domain techniques embed the watermark by updating the pixel values of the source image [4-5]. Transform domain techniques embed the watermark in the source image by means of updating the coefficients of the transformation domains such as discrete Fourier transformation (DFT), singular value decomposition (SVD), discrete wavelet transform (DWT) and discrete cosine transform (DCT) [6-7]. In general, transformation domain methods are more resistant against the attacks in comparison to spatial domain methods [8]. Numerous watermarking techniques have been proposed for images, videos and particularly the subjects of the invisibility of the watermark and its robustness against the attacks of the enemy have been considered important [9]. In robust watermarking algorithms, it is aimed that the information within the image to resist various image processing attacks and to be recognizable when it is recovered.

In [10], it has been proposed that the watermark becomes resistant when it is embedded in DC components in comparison to being embedded in AC components. Two predefined $\alpha$ scaling techniques have been used and evaluated accordingly to the block texture content.

In this study, the method recommended by Huang et. al. [10] is examined in detail. The effect of the scaling factor is expressed numerically and the robustness and transparency is analyzed by evaluating the DCT based watermarking technique, which modifies DC components, on different scaling factors and images.

\section{DCT BASED WATERMARKING USING DC COMPONENT}

DCT is a sinusoidal transform and similar to the Fourier transform however it only uses cosine terms and for that reason does not include complex components [11].

DCT is successfully used in many coding systems since it ensures the best energy distribution in the field of frequency [12].

A two-dimensional DCT is defined as follows [13]:

$F(u, v)=p(u) p(v) \sum_{x=0}^{M-1} \sum_{y=0}^{N-1} I(x, y) \cos \left(\frac{(2 x+1) u \pi}{2 M}\right)\left(\frac{(2 y+1) v \pi}{2 N}\right)$

where $I$ is the input image, $M$ and $N$ are the image height and width, respectively, $p(u)$ and $p(v)$ are calculated as follows: 


$$
p(k)= \begin{cases}\sqrt{\frac{1}{N}} & \text { if } k=0 \\ \sqrt{\frac{2}{N}} & \text { else }\end{cases}
$$

3. The watermark $w$ is created at random with a standard normal distribution as $\mu=0$ and $\sigma=1$

$$
\mathrm{W}=\left\{\mathrm{x}_{\mathrm{i}}, 0 \leq \mathrm{i} \leq \mathrm{k}\right\}
$$

In equation (3), $k$ is the number of blocks. The process of watermarking is realized only by updating the DC coefficients of each block. The embedding equation is as follows:

$$
\mathrm{F}_{\mathrm{k}}^{\prime}(\mathrm{u}, \mathrm{v})=\left\{\begin{array}{ll}
\mathrm{F}_{\mathrm{k}}(\mathrm{u}, \mathrm{v}) \cdot\left(1+\alpha \cdot \mathrm{x}_{\mathrm{k}}\right) & \text { if } \mathrm{u}=\mathrm{v}=0 \\
\mathrm{~F}_{\mathrm{k}}(\mathrm{u}, \mathrm{v}) & \text { otherwise }
\end{array}\right\}
$$

In equation (4), $\alpha$ is the scaling factor.

4. Inverse discreet cosine transform (IDCT) has been applied on the blocks and the watermarked image WI has been obtained.

\section{B. Watermark Extraction}

1. The watermarked image $W I$ and the original image $I$ have been decomposed into blocks of $8 \times 8$.

2. DCT is applied to all blocks individually.

3. The differences of DC components in each block of the original image and watermarked image are calculated as the watermark $w$.

$$
\begin{aligned}
& \mathrm{W}_{\mathrm{k}}^{*}=\mathrm{F}_{\mathrm{k}}^{*}(0,0)-\mathrm{F}_{\mathrm{k}}(0,0) \\
& \mathrm{W}^{*}=\bigcup_{\mathrm{i}} \mathrm{W}_{\mathrm{k}}^{*}\left\{\mathrm{x}_{\mathrm{i}}^{*}, 0 \leq \mathrm{i} \leq \mathrm{n}\right\}
\end{aligned}
$$

\section{EXPERIMENTAL RESULTS}

In robust watermarking it's aimed that to keep the level of degradation of the watermarked image as low as possible in comparison to the original picture (transparency) and to ensure that the watermark which has been extracted from the watermarked image as a result of attacks to resemble the original watermark as much as possible (robustness).

In this study, numerous experiments have been carried out using two grey level images with dimensions of $256 \times 256$ as shown in Figure 3. In the first case, host images are watermarked using different $\alpha$ values. It has been analyzed that to what extent the watermarked images have been degraded and to what extent the extracted images resembles to the original watermark. In another case, various attacks have been applied on watermarked images by using different $\alpha$ values and the robustness and transparency was analyzed.

Fig. 2. DCT based watermarking: embedding and extraction.

\section{A. Watermark Embedding}

1. The original image $I$, is decomposed into blocks $B$ of $8 \times 8$.

2. DCT is applied to all blocks individually. 


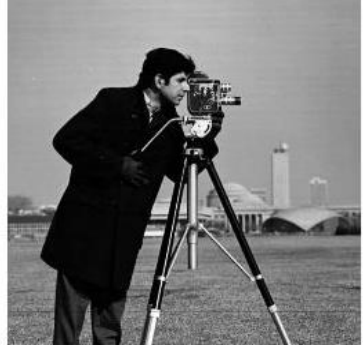

(a) Cameraman

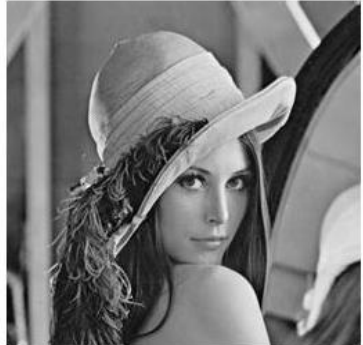

(b) Lena
Fig. 3. Original host images for watermarking experiments.

\section{A. Effect of the $\alpha$ parameter}

In the experiments implemented in this section, the effect of different $\alpha$ values between 0.005 and 0.030 has been analyzed. Host images that have been watermarked by different $\alpha$ values are shown in Figure 4. Only the results for $\alpha=0.005,0.015$ and 0,030 are shown due to the lack of space. When the visual results are evaluated, it has been seen that unwanted blocking effect arises in the watermarked images as the $\alpha$ value increases.

In Table 1, numerical results of correlation analysis are given to show that the similarity of the original watermark and the extracted watermark As seen from the Table 1, the degree of similarity of the extracted watermark to the original watermark increases as the scaling factor $(\alpha)$ increases.

In Table 2, transparency of the original host image and the watermarked image has been analyzed by means of PSNR values. Choosing a high scaling factor $(\alpha)$ causes degradation in the image.
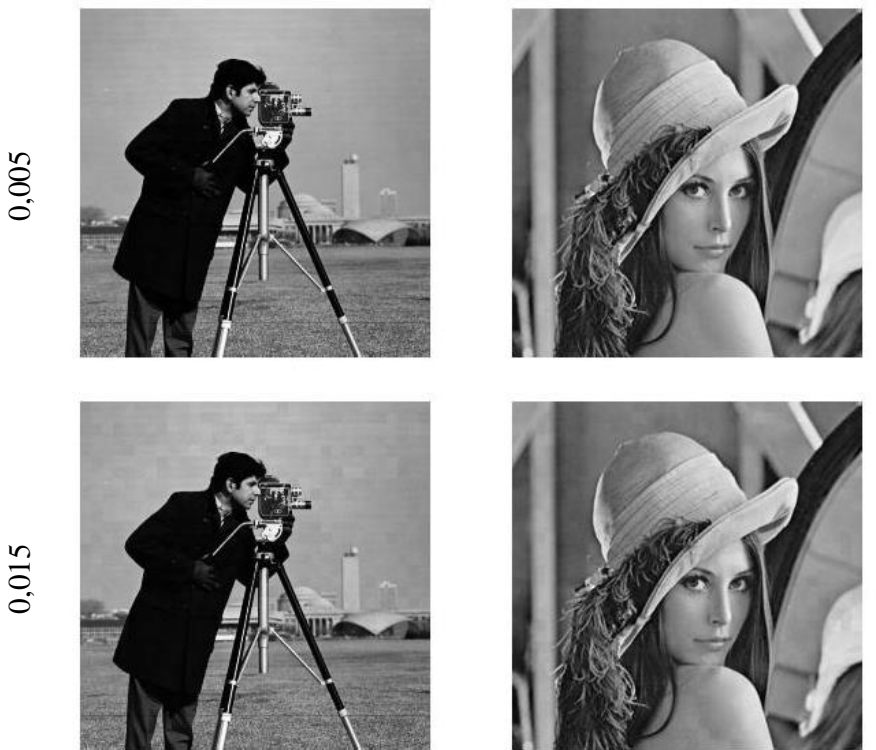
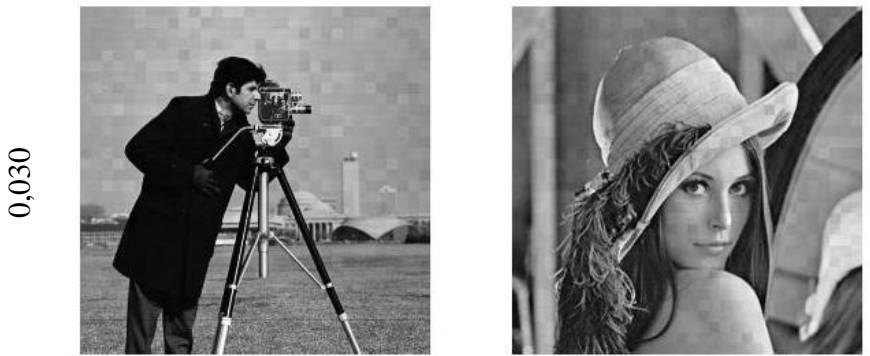

Fig. 4. Images watermarked with different $\alpha$ values.

TABLE I. ROBUSTNESS ANALYSIS FOR CAMERAMAN AND LENA ACCORDING TO SCALING FACTOR $(\alpha)$.

\begin{tabular}{|c|c|c|}
\hline CORR & Cameraman & Lena \\
\hline$\alpha=0.005$ & 0.8206 & 0.8368 \\
\hline$\alpha=0,010$ & 0.8857 & 0.9232 \\
\hline$\alpha=0,015$ & 0.8819 & 0.9364 \\
\hline$\alpha=0,020$ & 0.8936 & 0.9427 \\
\hline$\alpha=0,025$ & 0.9004 & 0.9390 \\
\hline$\alpha=0,030$ & 0.9037 & 0.9464 \\
\hline
\end{tabular}

B. Effect of Attacks and the a parameter

In this section, four different attacks have been carried out, namely blurring(BL), JPEG compression(JC), salt \& pepper noise(SP) and re-scaling(RS), for the purpose of testing the resistance of the watermarking method against attacks. Visual results and numerical analysis have been presented.

TABLE II. TRANSPARENCY ANALYSIS FOR CAMERAMAN AND LENA ACCORDING TO VARIOUS SCALING FACTORS $(\alpha)$.

\begin{tabular}{|c|c|c|}
\hline PSNR & Cameraman & Lena \\
\hline$\alpha=0.005$ & 117.2014 & 117.0920 \\
\hline$\alpha=0,010$ & 104.3893 & 104.3280 \\
\hline$\alpha=0,015$ & 95.9988 & 96.1121 \\
\hline$\alpha=0,020$ & 91.2254 & 90.5543 \\
\hline$\alpha=0,025$ & 87.1736 & 86.5733 \\
\hline$\alpha=0,030$ & 83.6518 & 82.3390 \\
\hline
\end{tabular}

Due to limited space, the attacks that have been applied to the watermarked image only for the $\alpha=0.015$ have been given for Cameraman and Lena, respectively, in the Figure 5 and 6.

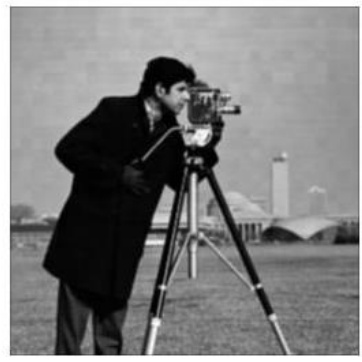

(a) Blurring

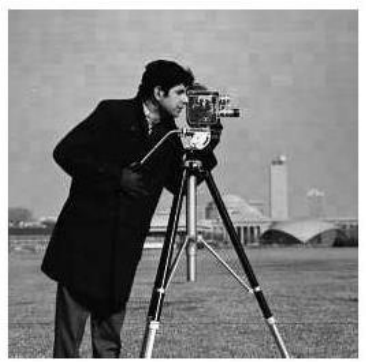

(b) Jpeg Compression 


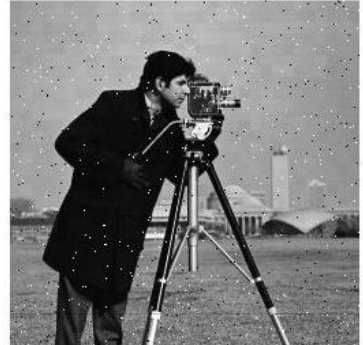

(c) Salt \& Pepper Noise

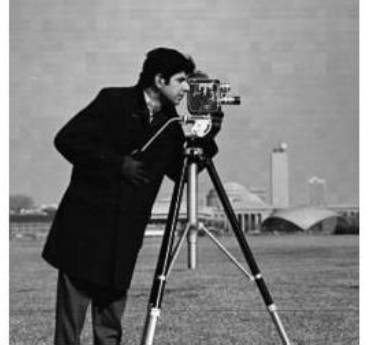

(d) Re-scaling

Fig. 5. Attacks on the watermarked images while $\alpha=0,015$ for Cameraman image.

In Table 3, the similarity of the watermarks, which have been extracted after various attacks, to the original watermark has been obtained by correlation values. For both of the test images, correlation values have been obtained as higher than 0.25 even at the lowest value of $\alpha=0.005$ and it was determined that the watermark is present in the image. On the other hand, the correlation values were over 0.8 and the existence of the watermark was undisputable at the value 0.030 .

\section{CONCLUSION}

In this study, effect of scaling parameter $(\alpha)$ on watermark extraction and degradation of image in robust watermarking of images has been examined by means of the modification of DC components in DCT. The effect of the watermarking method and $\alpha$ parameter has been analyzed by applying 4 different attacks. The results obtained have indicated that an appropriate $\alpha$ value must be chosen in order to ensure that the extracted watermark has a high degree of robustness and transparency.

\section{ACKNOWLEDGEMENTS}

The authors would like to thank Research Foundation of the Erciyes University, Kayseri, Turkey for supporting this work under the Grant No. FYL-2015-5826.

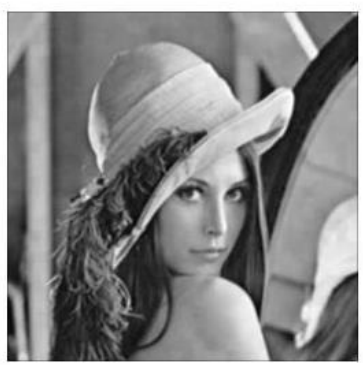

(a) Blurring

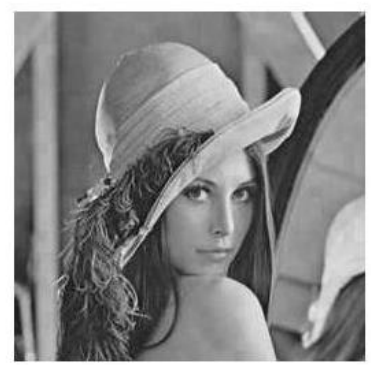

(b) Jpeg Compression

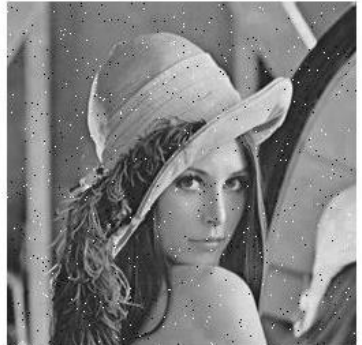

(c) Salt \& Pepper Noise

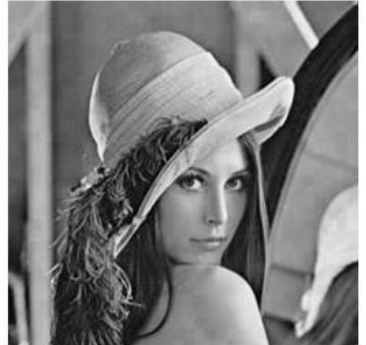

(d) Re-scaling
Fig. 6. Attacks on the watermarked images while $\alpha=0,015$ for Lena image.

TABLE III. ROBUSTNESS ANALYSIS AGAINST VARIOUS ATTACKS FOR CAMERAMAN AND LENA ACCORDING TO THE SCALING FACTOR

\begin{tabular}{|c|c|c|c|c|}
\hline CORR & \multicolumn{5}{|c|}{ Attacks } \\
\hline Cameraman & $\boldsymbol{B} \boldsymbol{L}$ & $\boldsymbol{J C}$ & $\boldsymbol{S P}$ & $\boldsymbol{R S}$ \\
\hline$\alpha=0.005$ & 0.2857 & 0.6545 & 0.2735 & 0.7949 \\
\hline$\alpha=0,010$ & 0.5479 & 0.8045 & 0.5474 & 0.8809 \\
\hline$\alpha=0,015$ & 0.6423 & 0.8475 & 0.6757 & 0.8779 \\
\hline$\alpha=0,020$ & 0.7217 & 0.8733 & 0.7190 & 0.8914 \\
\hline$\alpha=0,025$ & 0.7739 & 0.8864 & 0.7646 & 0.8978 \\
\hline$\alpha=0,030$ & 0.8127 & 0.8906 & 0.8231 & 0.9031 \\
\hline Lena & $\boldsymbol{B L}$ & $\boldsymbol{J C}$ & $\boldsymbol{S P}$ & $\boldsymbol{R S}$ \\
\hline$\alpha=0.005$ & 0.3443 & 0.6549 & 0.3164 & 0.8337 \\
\hline$\alpha=0,010$ & 0.5963 & 0.8405 & 0.5487 & 0.9231 \\
\hline$\alpha=0,015$ & 0.7213 & 0.9038 & 0.7276 & 0.9358 \\
\hline$\alpha=0,020$ & 0.8118 & 0.9174 & 0.8062 & 0.9424 \\
\hline$\alpha=0,025$ & 0.8338 & 0.9257 & 0.8264 & 0.9385 \\
\hline$\alpha=0,030$ & 0.8773 & 0.9390 & 0.8657 & 0.9466 \\
\hline
\end{tabular}

\section{REFERENCES}

[1] K. Prachi, L. Siddharth and T. Shreya, "Digital Watermarking for Protection of Intellectual Property", IJCEM International Journal of Computational Engineering \& Management, Vol. 12, April 2011, ISSN (Online): 2230-7893.

[2] G.C. Langelaar, I. Setyawan and R.L. Lagendijk, "Watermarking digital image and video data", IEEE Signal Processing Magazine, pp. 20-43, September 2000.

[3] C.S. Shieh, H.C. Huang, F.H. Wang, and J.S. Pan, "Genetic watermarking based on transform domain techniques", Pattern Recognition, vol. 37, pp. 555-565, 2004.

[4] Y.H. Yu, C.C Chang and Y.C. Hu, "Hiding secret data in images via predictive coding", Pattern Recognition, vol. 38, pp. 691-705, 2005.

[5] C.C. Chang, T.S. Chen and L.Z. Chung, "A steganographic method based upon JPEG and quantization table modification", Information Sciences, vol. 141, pp. 123-38, 2002.

[6] C.T. Hsu and J.L. Wu, "Multiresolution watermarking for digital images”, IEEE Trans Circuits Systems II: Analog Digital Signal Process, vol. 45, pp. 1097-101, 1998.

[7] G.J. Yu, C.S Lu and A. Mlhy, "Message-based cocktail watermarking system", Pattern Recognition, vol. 36, pp. 957-68, 2003

[8] V. Aslantas, "A singular value decomposition based image watermarking using genetic algorithm", Int J Electron Commun (AEU), 2007.

[9] Y. Xueyi, C. Xueting, D. Meng, H. Shuyun, W. Yunlu, "A MultipleLevel DCT Based Robust DWT-SVD Watermark Method," Computational Intelligence and Security (CIS), 2014 Tenth International Conference on, 2014, pp. 479-483 
ICIT 2015 The $7^{\text {th }}$ International Conference on Information Technology

doi:10.15849/icit.2015.0052 C ICIT 2015 (http://icit.zuj.edu.jo/ICIT15)

[10] J. Huang, Y.Q. Shi, Y. Shi, "Embedding image watermarks in DC components" (2000) IEEE Transactions on Circuits and Systems for Video Technology, 10 (6), pp. 974-979.

[11] Z. Pan and H. Bolouri, "High speed face recognition based on discrete cosine transforms and neural networks", University of Hertfordshire, 1999.

[12] E. Yen and L. Lin,"Rubik's cube watermark technology for grayscale images", Vol 37(6), pp 4033-4039, Jun. 2010.

[13] B. L. Gunjal, and R. R. Manthalkar, "An overview of transform domain robust digital image watermarking algorithms', Journal of Emerging Trends in Computing and Information Sciences, vol. 2, no 1, CIS Journal 2010-2011. 